OPEN ACCESS

Edited by:

Caroline Davis,

York University, Canada

Reviewed by:

Julia M. Hormes,

University at Albany, SUNY,

United States

Stephanie M. Manasse,

Drexel University, United States

*Correspondence:

Kelly C. Allison

kca@pennmedicine.upenn.edu

Specialty section: This article was submitted

to Eating Behavior,

a section of the journal

Frontiers in Nutrition

Received: 04 August 2017 Accepted: 08 November 2017 Published: 22 November 2017

Citation:

Chao AM, Srinivas SK, Studt SK,

Diewald LK, Sarwer DB and Allison KC (2017) A Pilot Randomized

Controlled Trial of a TechnologyBased Approach for Preventing Excess Weight Gain during Pregnancy among Women with Overweight. Front. Nutr. 4:57.

doi: 10.3389/fnut.2017.00057

\section{A Pilot Randomized Controlled Trial of a Technology-Based Approach for Preventing Excess Weight Gain during Pregnancy among Women with Overweight}

\author{
Ariana M. Chao ${ }^{1,2}$, Sindhu K. Srinivas ${ }^{3}$, Stacia K. Studt ${ }^{4}$, Lisa K. Diewald ${ }^{5}$, \\ David B. Sarwer ${ }^{6}$ and Kelly C. Allison ${ }^{2 *}$
}

${ }^{1}$ Department of Biobehavioral Health Sciences, University of Pennsylvania School of Nursing, Philadelphia, PA, United States, ${ }^{2}$ Department of Psychiatry, Perelman School of Medicine at the University of Pennsylvania, Philadelphia, PA, United States, ${ }^{3}$ Department of Obstetrics and Gynecology, Perelman School of Medicine at the University of Pennsylvania, Philadelphia, PA, United States, ${ }^{4}$ New York City Department of Health and Mental Hygiene, New York, NY, United States, ${ }^{5}$ Villanova University College of Nursing, Villanova, PA, United States, ${ }^{6}$ Center for Obesity Research and Education, Department of Social and Behavioral Science, Temple University College of Public Health, Philadelphia, PA, United States

Objective: Overweight/obesity and excess weight gain during pregnancy are associated with adverse maternal and neonatal outcomes. Few interventions have been effective in limiting gestational weight gain among women with overweight or obesity. This pilot, randomized clinical trial compared treatment as usual (TAU) to a lifestyle modification program delivered via phone for the prevention of excess gestational weight gain in women who had overweight or obesity.

Methods: Participants included 41 pregnant women with a body mass index $(\mathrm{BMl}) \geq 25 \mathrm{~kg} / \mathrm{m}^{2}$ (mean age $=28.7 \pm 5.8$ years; mean pre-gravid $\mathrm{BMl}=31.2 \pm$ $6.2 \mathrm{~kg} / \mathrm{m}^{2} ; 54 \%$ black, 39\% white). The intervention group ( $n=20$ ) received weekly telephone counseling sessions and used WiFi scales to monitor their weight from weeks 16 to 36 of pregnancy. We compared differences in weight and birth outcomes for the intervention vs. the TAU group $(n=21)$.

Results: The intervention and TAU groups did not differ with respect to: gestational weight gain $(15.5 \pm 5.3$ vs. $13.3 \pm 6.8 \mathrm{~kg}$, respectively); proportion gaining above the 2009 Institute of Medicine recommended weight range (83 vs. 70\%); and weight gain from pre-pregnancy weight to 6 weeks postpartum $(4.8 \pm 4.6$ vs. $3.0 \pm 5.5 \mathrm{~kg})$. Other birth and health outcomes also did not differ.

Conclusion: A telemedicine intervention designed to decrease logistical burden on participants was not more successful in reducing excessive weight gain during pregnancy as compared to TAU. Future studies should examine more intensive forms of remote treatment beginning earlier in pregnancy as well as interventions promoting a healthy weight prior to pregnancy.

Keywords: pregnancy, gestation, overweight, obesity, technology 


\section{INTRODUCTION}

Preventing excess gestational weight gain is one of the most significant modifiable risk factors that can help improve maternal and child health. Unfortunately, $47 \%$ of mothers in the United States typically exceed the amount of gestational weight gain recommended by the 2009 Institute of Medicine (IOM) guidelines (1). Of particular relevance are the recommended gestational weight ranges for women with pre-pregnancy overweight and obesity: $6.8-11.3 \mathrm{~kg}(15-25 \mathrm{lbs})$ for women with a body mass index (BMI) between 25 and $29.9 \mathrm{~kg} / \mathrm{m}^{2}$ (overweight); and 5.0 $9.1 \mathrm{~kg}(11-20 \mathrm{lbs})$ for women with a BMI of $30 \mathrm{~kg} / \mathrm{m}^{2}$ or greater (obesity) (2). Excess weight gain during pregnancy is associated with negative short- and long-term health consequences for both mothers and their children including gestational diabetes mellitus, preeclampsia, postpartum weight retention, congenital abnormalities, stillbirth, and fetal macrosomia (3-7). Over 50\% of women entering pregnancy can be classified as overweight or obese (8). These women are particularly vulnerable to excess gestational weight gain, with double the risk of gaining more than the recommended amount of weight during pregnancy compared to women who are of normal weight (9).

Pregnancy may be an opportune time for weight management since many women have regular contacts with health-care professionals and are potentially more motivated to maintain a healthy lifestyle $(10,11)$. However, there are several challenges to successful antenatal weight management. Barriers often cited by pregnant women revolve around pragmatic issues associated with attending a weight management program, including a lack of time to attend sessions due to competing demands and geographical constraints that may hinder the receipt of services $(12,13)$. Health-care providers also encounter significant barriers to providing weight management, such as lack of training in delivering weight counseling (14) and minimal time during appointments to discuss these issues (15). Results from interventions to prevent excessive gestational weight gain among women who have overweight or obesity have been modest. In a recent meta-analysis, pregnant women with overweight or obesity who were enrolled in lifestyle interventions gained an average of $1.7 \mathrm{~kg}$ less than control groups (16). Taking these challenges together, including the modest outcomes and logistical challenges documented thus far, low burden, effective, and scalable weight management interventions are needed for this population.

Technology-based interventions delivered in a woman's home can help decrease the logistical constraints and burden common with weight management interventions $(17,18)$. Telemedicine is a technology-based approach that has become increasingly popular due to its high potential to be integrated into clinical practice and capacity to maintain patient-provider contact. It can include telephone counseling, text messages, web-based programs, and other wearable and WiFi devices that monitor weight and activity. In non-pregnant samples, telephone counseling produces similar weight loss compared to in-person counseling $(19,20)$. Of all the telehealth approaches, it seems that telephone counseling may provide the highest treatment intensity to optimize weight outcomes. Counseling visits can be combined with newer technologies such as "smart" scales to help ensure that important elements of weight-loss counseling are not omitted (21). These in-home body-weight scales transmit a patient's weight to a clinician via WiFi. Health-care providers can then provide women with tailored feedback based on objective measures. "Smart" scales have been effective in obesity treatment interventions in non-pregnant samples (22), but the outcomes of this approach during pregnancy are not known. Little is known about the efficacy of this strategy in limiting excessive gestational weight gain.

The purpose of this pilot, randomized controlled trial was to test the feasibility and preliminary efficacy of an intervention consisting of telephone counseling focused on limiting excess weight gain during pregnancy plus WiFi weighing compared to a treatment as usual (TAU) control group. The primary aim was to compare the amount of weight gained during pregnancy among women in the intervention group as compared to women who received TAU. The hypothesis was that women in the intervention group would gain significantly less weight than those in the TAU. We also hypothesized that a smaller proportion of women in the intervention group would exceed the IOM's recommended ranges for weight gain. An exploratory aim was to compare caloric intake among women in the intervention group as compared to women in the TAU group. We hypothesized that women in the intervention group would consume fewer total calories. Finally, we described the acceptability of the intervention and the usefulness of each of its components, as rated by participants.

\section{MATERIALS AND METHODS}

\section{Participants and Design}

Women who were up to 16 weeks pregnant were recruited from two obstetrics clinics in Philadelphia, PA, USA. Inclusion criteria were self-reported pre-pregnancy BMI between 25 and $50 \mathrm{~kg} / \mathrm{m}^{2}$; 18 and 40 years of age; and ability to read and understand English. Exclusion criteria were diabetes mellitus; history of gestational diabetes; twins or other multiples; HIV; and chronic steroid use. Women were screened at the clinics, self-reported pre-pregnancy height and weights were recorded, and pre-pregnancy BMI was calculated. Participants' electronic medical records were accessed to obtain the mother's weight at gestational age of 16 weeks (or weight closest to that age; range 13-16 weeks). The women provided written informed consent in accordance with the Declaration of Helsinki if they reached the inclusion criteria and were enrolled in the study after they completed a baseline questionnaire with contact and demographic information, and information about their eating habits and exercise routines. This study was reviewed and approved by the University of Pennsylvania Institutional Review Board.

\section{Intervention}

The intervention consisted of weekly, 20-min telephone counseling sessions with a dietician between weeks 16 and 36 gestation or delivery of their baby, whichever came first. The dietician was trained in behavioral weight-loss treatment. A treatment manual based on the Look AHEAD trial was 
adapted from a behavioral weight-loss intervention to a behavioral weight management intervention focusing on appropriate gestational weight gain (23). The sessions addressed the domains associated with weight control: nutrition, exercise, and lifestyle modification strategies to improve adherence to the diet and activity plan, decrease disordered eating and response to environmental food cues, and improve mood and stress. Participants also kept food records to help them stay within recommended calorie ranges, aiming for an increase of only 300 cal per day above pre-pregnancy weight maintenance levels. As recommended in the IOM guidelines (2), weight gain goals during the second and third trimesters were $0.6 \mathrm{lbs} /$ week for women with overweight and $0.5 \mathrm{lbs} /$ week for women with obesity. These calculations assumed a 1.1- to 4.4-lb gain in the first trimester and were adjusted in each participant's weight gain charts according to her actual first trimester gains. The women in this group were asked to weigh themselves weekly with a WiFi scale (www.Withings.com). The WiFi scales transmitted their weights to personalized weight charts that could be accessed via the Internet by the participant and study staff for remote weight monitoring and to give feedback to participants. During each weekly phone call, the study dietician reviewed a treatment session and gave individualized feedback based on the measured weights, verbal review of the food records, and any other adherence issues that the participants presented.

The TAU group received the counseling typically provided at their obstetrics visits on nutrition, exercise, and weight gain goals (24). They did not receive the telephone and WiFi weighing intervention, or specific feedback from study staff on the course of weight gain during their pregnancies. All women who were enrolled in the study completed monthly, 24-h food recalls using the Internet-based Automated Self-administered 24-h Recall (ASA24) (25).

\section{Measures}

\section{Demographic and Clinical Information}

Participants completed a baseline survey that included questions on age, race, marital status, education, pregnancy history, and current smoking status.

\section{Weight and Birth Outcomes}

Pre-pregnancy weight was self-reported. For participants who had a weight recorded in the electronic medical record in the year prior to their pregnancy (TAU, $n=9$ and intervention, $n=9$ ), the mean difference between self-reported and measured weight was $1.8 \pm 13.5 \mathrm{lbs}$. A structured medical abstraction form was used to extract data from participants' electronic medical records. Final pregnancy weight was taken from the last measured weight during participants' prenatal visits. Maternal and neonatal birth outcomes and maternal weight at 6 weeks postpartum, where available, were also extracted.

\section{Questionnaires}

Caloric intake was calculated from 24-h food records that were completed on the internet (25). Additional questionnaires were administered at baseline to provide a more comprehensive picture of these participants' eating patterns, sleep duration, mood, and physical activity. Night eating was assessed using the Night Eating Questionnaire, a 14-item validated measure of night eating syndrome (NES) (26). All items, except one used to screen for the parasomnia sleep-related eating disorder, were scored on a 0 to 4 Likert scale and summed to obtain a global score. The NEQ has a possible range of $0-54$ with higher scores indicating more NES symptoms; a score $\geq 25$ is suggestive of NES. The Cronbach's alpha in this sample was 0.66. Binge eating was measured using self-reported questions from the Eating Disorder Examination-Questionnaire (27). The items asked individuals if they consumed an unusually large amount of food while feeling a sense of loss control over eating during the past 28 days as well as the number of episodes over the past 4 weeks. We assessed average hours of sleep per night using the Pittsburgh Sleep Quality Index (28). Participants completed the 10-item Edinburgh Postnatal Depression Scale (EPDS) (29). Items were scored on a 0 to 3 Likert scale and summed to generate a total score. Higher scores indicated more depressive symptoms and scores of $\geq 10$ indicate a moderate to high risk of perinatal of depression. The Cronbach's alpha in this sample was 0.88 . Stress was assessed using the 4 -item Perceived Stress Scale (PSS) with a possible range of $0-15$ and higher scores indicating more stress (30). Norms suggest a typical score is $6.4(\mathrm{SD}=3.1)$ for women $(31)$. The Cronbach's alpha was 0.65 . At baseline, participants were asked about the amount of time spent each week on physical activity (i.e., sports, leisure, or recreational activities) since becoming pregnant. At week 36, participants in the intervention group completed questions that assessed how helpful the intervention was to them. Scores ranged from 1 (did not help at all) to 5 (very helpful).

\section{Statistical Analysis Power Analysis}

The proposed study was powered to detect differences between groups for the primary aim. If the Intervention group limited weight gain to $7 \mathrm{~kg}(\mathrm{SD}=5.5)$, as it was in a previous study by Wolff et al. (32) and the TAU group gained $14.3 \mathrm{~kg}(\mathrm{SD}=9.9)$, as they did in the investigators' previous study (33), then 21 participants per group would yield $80 \%$ power, using a two group Satterthwaite $t$-test with a 0.05 two-sided alpha level (nQuery Advisor, version 6). If the Intervention group gained $8 \mathrm{~kg}$, then 28 participants per group would yield $80 \%$ power. Given the short time-constraints of the study, we proposed to recruit 21 participants per group.

Data were analyzed using SPSS version 24.0. Descriptive statistics were used to characterize the sample (i.e., means, SDs, percentages). Independent sample $t$-tests and chi-square tests were conducted to compare the intervention and TAU groups on all main outcome measures. Pearson correlations were used to assess the relationship between intervention session attendance and weight loss. We modeled caloric intake over the intervention period using linear mixed models. A $p$-value $<0.05$ was considered statistically significant. 


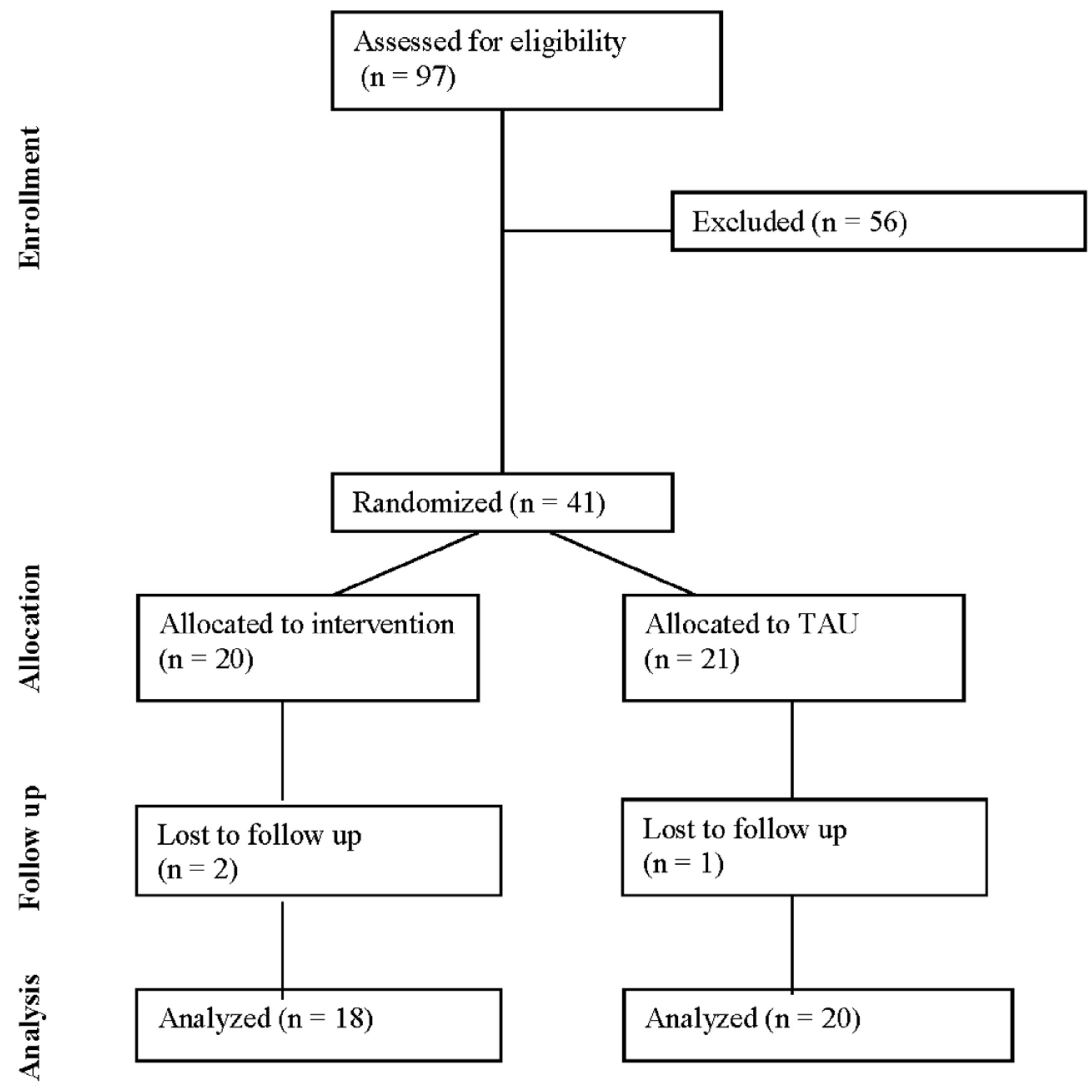

FIGURE 1 | CONSORT diagram.

\section{RESULTS}

The CONSORT diagram for this study is presented in Figure 1. In the total sample $(n=41)$, participants had a mean age of $28.7 \pm 5.8$ years and the sample was $53.7 \%$ black, $39.0 \%$ white, $7.2 \%$ other. Approximately half (51.2\%) of participants were married and $48.8 \%$ had graduated from college. One participant was a current smoker. Mean gravidity (i.e., the number of times a woman has been pregnant) was $1.3 \pm 1.5$ and parity (i.e., the number of times a woman has given birth to a fetus of 24 weeks or more) was $0.8 \pm 1.0$. Participants had a mean pregestational BMI of $31.1 \pm 6.2 \mathrm{~kg} / \mathrm{m}^{2}$ with $53.7 \%$ of participants beginning the pregnancy in the overweight range and $46.3 \%$ reporting a pregestational $\mathrm{BMI}$ in the obese range. On average, scores on the EPDS were $6.6 \pm 5.6$ with 10 (24.4\%) women at moderate to high risk of perinatal depression. The mean PSS score was $5.7 \pm 3.8$, which was within a normal range, and NEQ score was $14.5 \pm 6.7$, suggesting the presence of subthreshold night eating symptoms. Participants reported an average of $7.1 \pm 1.5 \mathrm{~h}$ of sleep per night. One participant reported binge eating (two episodes in the past 4 weeks) at baseline. Participants reported engaging in an average of $46.5 \pm 91.8$ min of physical activity per week with $68.3 \%$ reporting no weekly physical activity. Baseline characteristics were not significantly different between the intervention $(n=20)$ and treatment $(n=21)$ groups ( $p s>0.05$; Table 1$)$.
TABLE 1 | Baseline demographic and clinical characteristics by treatment arm.

\begin{tabular}{lccc}
\hline & $\begin{array}{c}\text { Intervention } \\
(\boldsymbol{n}=\mathbf{2 0}) \text { mean } \\
\text { (SD) or } \boldsymbol{N}(\%)\end{array}$ & $\begin{array}{c}\text { TAU }(\boldsymbol{n}=\mathbf{2 1}) \\
\text { mean (SD) or } \\
\boldsymbol{N}(\%)\end{array}$ & p-Value \\
\hline Age, years & $29.1(6.4)$ & $28.4(5.2)$ & 0.73 \\
Race & & & \\
Black & $9(45.0)$ & $13(61.9)$ & 0.36 \\
White & $10(50.0)$ & $6(28.6)$ & \\
Other & $1(5.0)$ & $2(9.5)$ & \\
Married & $12(60.0)$ & $9(42.9)$ & 0.35 \\
Education & & & \\
$\quad$ Not college graduate & $10(50.0)$ & $11(52.4)$ & 0.88 \\
$\quad$ College graduate & $10(50.0)$ & $10(47.6)$ & \\
Gravidity & $1.0(1.1)$ & $1.7(1.7)$ & 0.11 \\
Parity & $0.8(1.0)$ & $0.9(1.1)$ & 0.63 \\
Pregestational BMI (kg/m²) & $31.0(4.8)$ & $31.3(7.5)$ & 0.90 \\
Pregestational BMI status & & & \\
$\quad$ Overweight & $10(50.0)$ & $12(57.1)$ & 0.76 \\
$\quad$ Obese & $10(50.0)$ & $9(42.9)$ & \\
Depressive symptoms (EPDS) & $6.9(4.4)$ & $6.3(6.6)$ & 0.75 \\
Perceived stress scale (PSS) & $6.2(2.9)$ & $5.1(4.5)$ & 0.37 \\
Night eating (NEQ) & $15.4(6.5)$ & $13.6(7.0)$ & 0.40 \\
Sleep per night, hours (PSQl) & $7.3(1.7)$ & $6.9(1.2)$ & 0.36 \\
\hline
\end{tabular}

TAU, treatment as usual; EPDS, Edinburgh Postnatal Depression Scale; PSS, Perceived Stress Scale; NEQ, Night Eating Questionnaire; PSQI, Pittsburgh Sleep Quality Index; BMI, body mass index. 
Before the intervention began, the amount of weight gain in the first 16 weeks was $4.8 \pm 4.6 \mathrm{~kg}$ in the intervention group and $3.0 \pm 5.5 \mathrm{~kg}(p=0.33)$ in the TAU group. Total gestational weight gain did not differ significantly $(p=0.29)$ between the intervention $(15.5 \pm 5.3 \mathrm{~kg}$; range: $4.5-25.4 \mathrm{~kg})$ and TAU $(13.3 \pm 6.8 \mathrm{~kg}$; range: -1.8 to $28.6 \mathrm{~kg})$ groups. The Cohen's $d$ for gestational weight gain was 0.36 . The proportion of the sample exceeding IOM weight gain recommendations was high in both the intervention (83.3\%) and TAU (70\%, $p=0.74)$ groups. The intervention and TAU groups did not differ in change in reported caloric intake over the course of the intervention $(2,154 \pm 251.3$ vs. $1,972.0 \pm 202.7$ kcals; $p=0.58)$.

Participants in the telemedicine group completed a mean of $12.5 \pm 6.4$ telephone counseling sessions. The number of intervention sessions completed inversely correlated with pre-pregnancy BMI $(r=-0.46, p=0.04)$ and positively with gestational weight gain $(r=0.55, p=0.02)$. Internet access varied throughout the study, with $58 \%$ having access across the entire length of the intervention. Of the 14 intervention participants who completed the week 36 assessment, most participants rated the intervention components as helpful in affecting pregnancy weight gain (Table 2). Participants rated talking with the dietician as most helpful $(4.4 \pm 0.9)$, followed by weekly weighing on the scale $(4.3 \pm 0.8)$ and sharing their weight each week with the study staff $(4.1 \pm 0.8)$.

Sixteen participants in the intervention group and 16 in the TAU group had data available on weight change from prepregnancy to 6-week postpartum, which did not differ between groups $(4.8 \pm 4.6$ vs. $3.0 \pm 5.5 \mathrm{~kg}$, respectively, $p=0.33)$. The intervention and TAU group did not differ in their 1-h $50 \mathrm{~g}$ glucose test results $(116.0 \pm 35.3$ vs. $113.9 \pm 23.2 \mathrm{mg} / \mathrm{dL}, p=0.82)$. Gestational week at delivery did not differ between the intervention $(38.6 \pm 1.8)$ and TAU groups $(39.4 \pm 1.3 ; p=0.10)$. The intervention and TAU groups did not differ in the birth weight $(3,254.6 \pm 653.1$ vs. $3,381.1 \pm 552.9 \mathrm{~g}, p=0.52)$ or 5 -min Apgar scores $(8.8 \pm 0.7$ vs. $8.9 \pm 0.5, p=0.73)$ of their babies.

\section{DISCUSSION}

In this preliminary efficacy study, a telephone-based approach combined with WiFi weighings was not able to decrease excess gestational weight gain among this diverse sample of women with overweight or obesity. The majority of the sample gained more

TABLE 2 | Intervention process rating scores $(n=14)$

\begin{tabular}{ll}
\hline & Mean (SD) \\
\hline Stepping on scale each week & $4.29(0.83)$ \\
Sharing your weight each week with study staff & $4.07(0.83)$ \\
Talking with the dietician each week & $4.36(0.93)$ \\
Using materials in the workbook & $3.50(1.35)$ \\
Conversing with your obstetrician and other medical care providers & $2.36(1.39)$ \\
Keeping daily, paper food logs & $4.00(0.96)$ \\
Completing internet-based 24-h food recalls & a \\
\end{tabular}

Scores range from 1 (did not help at all) to 5 (very helpful) in affecting pregnancy weight gain.

aNine participants completed this question. than the 2009 IOM guidelines for recommended weight gain, and birth outcomes did not differ between groups. A small number of studies have tested the efficacy of technology-based interventions among samples of pregnant women with overweight or obesity. Some studies have shown that technology-based interventions can result in minimal to modest benefits in gestational weight gain $(17,18)$. However, this technology-based intervention yielded no benefits for limiting excessive gestational weight gain as compared to TAU. Previous weight management interventions have been more successful in controlling weight gain among women of normal weight, but not women with overweight or obesity [see Herring et al. (34) for review]. Some factors may be the truncated recommended weight gain ranges for these groups, reduced activity levels, and/or poorer diet quality.

In both groups, there was clinically significant weight gain before the intervention started at 16 weeks. Many women gain much of their pregnancy weight during the first trimester (35). Early gestational weight gain is also predictive of subsequent weight gain (36). With the IOM's recommended upper limit of $11.3 \mathrm{~kg}$ (25 lbs) for women with overweight and $9.1 \mathrm{~kg}$ (20 lbs) for women with obesity, our intervention group was about halfway to this mark by 16 weeks. The $1.8 \mathrm{~kg}$ ( $4 \mathrm{lbs}$ ) difference between the intervention and TAU groups at week 16 seemed to follow the groups at delivery and at 6 weeks postpartum. Therefore, it is likely that interventions focused on preventing excessive gestational weight gain should start earlier, and ideally before conception, as recommended by the IOM (2). The strategy of treating overweight/obesity with lifestyle modification prior to conception has been successful in improving outcomes among women with polycystic ovarian syndrome (PCOS), a common endocrine condition affecting women of reproductive age $(37,38)$. Future studies are necessary to assess the efficacy of this approach on maternal and neonatal outcomes in women with overweight/obesity without PCOS.

In this study, telephone sessions were a feasible way to deliver weight-loss counseling among pregnant women. The WiFi scales relied on cellular service and a significant number of participants did not have reliable WiFi service to use such scales. Not having access to reliable and high-speed internet could reduce feasibility and pose barriers when implementing these technologies. In the current study, participants self-reported the weight on their Withings scales to the dietician, and the weights were entered manually on the participants' Internet-based weight charts.

It is possible that the WiFi weighing and lifestyle counseling telephone sessions could improve outcomes if combined with other methods for weight control. For example, telephone counseling sessions have been most frequently used with in-person visits and have been an effective strategy in some $(39,40)$, but not all studies (41-43). Several other technologies have been used to address gestational weight gain with varying success including websites, mobile phone applications, text messaging, email, and video (18). A recent study combined several of these approaches to decrease postpartum weight retention among women with overweight/ obesityrecruited throughtheWomen, Infant, andChildren program (44). Women in the intervention were significantly more likely to return to pre-gravid weight at 6 months postpartum, as compared to the usual care group. Another potential direction is integrating 
technology to improve self-monitoring and to allow for more intensive monitoring by healthcare providers. Self-monitoring of weight, eating behaviors, and physical activity is a crucial part of weight loss as well as maintenance. WiFi scales were provided as part of the intervention to help patients monitor their weight as well as to allow interventionists to provide feedback to patients remotely. Participants viewed the scales as helpful. Incorporating other technologies to improve self-monitoring of food intake (e.g., food monitoring apps) and physical activity (e.g., wearable activity trackers) may help to promote better outcomes. Evidence is currently inconclusive in technology-based interventions for weight management during pregnancy, and more research is needed to determine the ideal treatment components, delivery, and intensity.

Limitations to this study include a small sample size with limited statistical power, use of self-reported pregestational weight, and short-term follow-up. We relied on self-reported pre-pregnancy weights which can lead to inaccurate weight classification. Using the subset of participants with available pre-pregnancy weights, we found that the mean difference was less than $0.8 \mathrm{~kg}$ (1.8 lbs), but that variability was wide. In addition, physical activity was not tracked or monitored in this study. Use of 24-h recalls could have resulted in some reactivity among the women in the TAU group. However, this method of dietary assessment is considered to be one of the least biased among selfreport methods in terms of reactivity (45).

In conclusion, an intervention that included behavioral nutrition counseling via phone combined with WiFi measures of weight was not effective in reducing excess gestational weight gain among overweight and obese pregnant women. We hypothesize that starting an intervention program earlier or having a more intensive program might yield a better impact overall. Future research is needed to determine the optimal time

\section{REFERENCES}

1. Deputy NP, Sharma AJ, Kim SY, Hinkle SN. Prevalence and characteristics associated with gestational weight gain adequacy. Obstet Gynecol (2015) 125(4):773-81. doi:10.1097/AOG.0000000000000739

2. Institute of Medicine and National Research Council Committee to Reexamine IOM Pregnancy Weight Guidelines. Weight Gain during Pregnancy: Reexamining the Guidelines. Washington, DC: National Academies Press (2009).

3. Kiel DW, Dodson EA, Artal R, Boehmer TK, Leet TL. Gestational weight gain and pregnancy outcomes in obese women: how much is enough? Obstet Gynecol (2007) 110(4):752-8. doi:10.1097/01.AOG.0000278819.17190.87

4. Jolly MC, Sebire NJ, Harris JP, Regan L, Robinson S. Risk factors for macrosomia and its clinical consequences: a study of 350,311 pregnancies. Eur J Obstet Gynecol Reprod Biol (2003) 111(1):9-14. doi:10.1016/S0301-2115(03)00154-4

5. Siega-Riz AM, Viswanathan M, Moos MK, Deierlein A, Mumford S, Knaack J, et al. A systematic review of outcomes of maternal weight gain according to the Institute of Medicine recommendations: birthweight, fetal growth, and postpartum weight retention. Am JObstet Gynecol (2009) 201(4):339.e1-14. doi:10.1016/j.ajog.2009.07.002

6. Khashan AS, Kenny LC. The effects of maternal body mass index on pregnancy outcome.EurJ Epidemiol(2009)24(11):697-705.doi:10.1007/s10654-009-9375-2

7. Sarwer DB, Allison KC, Gibbons LM, Markowitz JT, Nelson DB. Pregnancy and obesity: a review and agenda for future research. J Womens Health (2006) 15(6):720-33. doi:10.1089/jwh.2006.15.720

8. National Center for Health Statistics (US). Health, United States, 2015: With Special Feature on Racial and Ethnic Health Disparities. Hyattsville, MD: National Academies Press (2016). to start such an intervention, as well as the components and method of delivery.

\section{ETHICS STATEMENT}

The women provided written informed consent in accordance with the Declaration of Helsinki if they reached the inclusion criteria and were enrolled in the study after they completed a baseline questionnaire with contact and demographic information and information about their eating habits and exercise routines. This study was reviewed and approved by the University of Pennsylvania Institutional Review Board.

\section{AUTHOR CONTRIBUTIONS}

KA, SSrinivas, and DS made substantial contributions to the conception or design of the work; AC, SSrinivas, SStudt, LD, DS, and KA contributed to the acquisition, analysis, or interpretation of data for the work. All authors participated in the drafting the work or revising it critically for important intellectual content, final approval of the version to be published, and agreement to be accountable for all aspects of the work in ensuring that questions related to the accuracy or integrity of any part of the work are appropriately investigated and resolved.

\section{FUNDING}

This work was supported by a GlaxoSmithKline Research grant from The Obesity Society (PI: KA). AC was funded by a postdoctoral fellowship from the National Institutes of Nursing Research/ NIH (T32NR007100-17). The contents of this work are solely the responsibility of the authors and do not necessarily represent the official view of NIH.

9. Restall A, Taylor RS, Thompson JM, Flower D, Dekker GA, Kenny LC, et al. Risk factors for excessive gestational weight gain in a healthy, nulliparous cohort. J Obes (2014) 2014:148391. doi:10.1155/2014/148391

10. Clissold T, Hopkins W, Seddon R. Lifestyle behaviours during pregnancy. N Z Med J (1991) 104(908):111-2.

11. Phelan S. Pregnancy: a "teachable moment" for weight control and obesity prevention. Am J Obstet Gynecol (2010) 202(2):135.e1-8. doi:10.1016/j. ajog.2009.06.008

12. Olander EK, Atkinson L. Obese women's reasons for not attending a weight management service during pregnancy. Acta Obstet Gynecol Scand (2013) 92(10):1227-30. doi:10.1111/aogs.12195

13. Sui Z, Turnbull D, Dodd J. Overweight and obese women's perceptions about making healthy change during pregnancy: a mixed method study. Matern Child Health J (2013) 17(10):1879-87. doi:10.1007/s10995-012-1211-8

14. Macleod M, Gregor A, Barnett C, Magee E, Thompson J, Anderson AS. Provision of weight management advice for obese women during pregnancy: a survey of current practice and midwives' views on future approaches. Matern Child Nutr (2013) 9(4):467-72. doi:10.1111/j.1740-8709.2011.00396.x

15. Heslehurst N, Newham J, Maniatopoulos G, Fleetwood C, Robalino S, Rankin J. Implementation of pregnancy weight management and obesity guidelines: a meta-synthesis of healthcare professionals' barriers and facilitators using the Theoretical Domains Framework. Obes Rev (2014) 15(6):462-86. doi:10.1111/obr.12160

16. Yeo S, Walker JS, Caughey MC, Ferraro AM, Asafu-Adjei JK. What characteristics of nutrition and physical activity interventions are key to effectively reducing weight gain in obese or overweight pregnant women? A systematic review and meta-analysis. Obes Rev (2017) 18(4):385-99. doi:10.1111/obr.12511 
17. O'Brien O, McCarthy M, Gibney E, McAuliffe F. Technology-supported dietary and lifestyle interventions in healthy pregnant women: a systematic review. Eur J Clin Nutr (2014) 68(7):760-6. doi:10.1038/ejcn.2014.59

18. Lau Y, Klainin-Yobas P, Htun T, Wong SN, Tan KL, Ho-Lim ST, et al. Electronic-based lifestyle interventions in overweight or obese perinatal women: a systematic review and meta-analysis. Obes Rev (2017) 18(9): 1071-87. doi:10.1111/obr.12557

19. Donnelly JE, Smith BK, Dunn L, Mayo MM, Jacobsen DJ, Stewart EE, et al. Comparison of a phone vs clinic approach to achieve $10 \%$ weight loss. Int J Obes (2007) 31(8):1270-6. doi:10.1038/sj.ijo.0803568

20. Appel LJ, Clark JM, Yeh HC, Wang NY, Coughlin JW, Daumit G, et al. Comparative effectiveness of weight-loss interventions in clinical practice. N Engl J Med (2011) 365(21):1959-68. doi:10.1056/NEJMoa1108660

21. Zheng Y, Klem ML, Sereika SM, Danford CA, Ewing LJ, Burke LE. Selfweighing in weight management: a systematic literature review. Obesity (2015) 23(2):256-65. doi:10.1002/oby.20946

22. Steinberg DM, Tate DF, Bennett GG, Ennett S, Samuel-Hodge C, Ward DS. The efficacy of a daily self-weighing weight loss intervention using smart scales and e-mail. Obesity (2013) 21(9):1789-97. doi:10.1002/oby.20396

23. Look AHEAD Research Group, Wadden TA, West DS, Delahanty L, Jakicic J, Rejeski J, et al. The look AHEAD study: a description of the lifestyle intervention and the evidence supporting it. Obesity (2006) 14(5):737-52. doi:10.1038/ oby.2006.84

24. American College of Obstetricians and Gynecologists Committee on Health Care for Underserved Women. Challenges for overweight and obese women. Obstet Gynecol (2014) 123(3):726-30. doi:10.1097/01.AOG.0000444457. 29401.a0

25. Subar AF, Crafts J, Zimmerman TP, Wilson M, Mittl B, Islam NG, et al. Assessment of the accuracy of portion size reports using computer-based food photographs aids in the development of an automated selfadministered 24-hour recall. J Am Diet Assoc (2010) 110(1):55-64. doi:10.1016/ j.jada.2009.10.007

26. Allison KC, Lundgren JD, O'Reardon JP, Martino NS, Sarwer DB, Wadden TA, et al. The Night Eating Questionnaire (NEQ): psychometric properties of a measure of severity of the Night Eating Syndrome. Eat Behav (2008) 9(1):62-72. doi:10.1016/j.eatbeh.2007.03.007

27. Fairburn CG, Beglin SJ. Assessment of eating disorders: interview or selfreport questionnaire? Int J Eat Disord (1994) 16(4):363-70.

28. Buysse DJ, Reynolds CF III, Monk TH, Berman SR, Kupfer DJ. The Pittsburgh Sleep Quality Index: a new instrument for psychiatric practice and research. Psychiatry Res (1989) 28(2):193-213. doi:10.1016/0165-1781(89) 90047-4

29. Cox JL, Holden JM, Sagovsky R. Detection of postnatal depression. Development of the 10-item Edinburgh Postnatal Depression Scale. Br J Psychiatry (1987) 150(6):782-6. doi:10.1192/bjp.150.6.782

30. Cohen S, Williamson G. Perceived stress in a probability sample of the U.S. In: Spacapam S, Oskamp S, editors. The Social Psychology of Health: Claremont Symposium on Applied Social Psychology. Newbury Park, CA: Sage (1988).

31. Warttig SL, Forshaw MJ, South J, White AK. New, normative, English-sample data for the short form perceived stress scale (PSS-4). J Health Psychol (2013) 18(12):1617-28. doi:10.1177/1359105313508346

32. Wolff S, Legarth J, Vangsgaard K, Toubro S, Astrup A. A randomized trial of the effects of dietary counseling on gestational weight gain and glucose metabolism in obese pregnant women. Int J Obes (2008) 32(3):495-501. doi:10.1038/sj.ijo.0803710

33. Allison KC, Wrotniak BH, Paré E, Sarwer DB. Psychosocial characteristics and gestational weight change among overweight, African American pregnant women. Obstet Gynecol Int (2012) 2012:9. doi:10.1155/2012/878607
34. Herring SJ, Rose MZ, Skouteris H, Oken E. Optimizing weight gain in pregnancy to prevent obesity in women and children. Diabetes Obes Metab (2012) 14(3):195-203. doi:10.1111/j.1463-1326.2011.01489.x

35. Jersey SJ, Nicholson J, Callaway LK, Daniels LA. A prospective study of pregnancy weight gain in Australian women. Aust N Z J Obstet Gynaecol (2012) 52(6):545-51. doi:10.1111/ajo.12013

36. Carreno CA, Clifton RG, Hauth JC, Myatt L, Roberts JM, Spong CY, et al. Excessive early gestational weight gain and risk of gestational diabetes mellitus in nulliparous women. Obstet Gynecol (2012) 119(6):1227-33. doi:10.1097/AOG.0b013e318256cfla

37. Legro RS, Dodson WC, Kunselman AR, Stetter CM, Kris-Etherton PM, Williams NI, et al. Benefit of delayed fertility therapy with preconception weight loss over immediate therapy in obese women with PCOS. JClin Endocrinol Metab (2016) 101(7):2658-66. doi:10.1210/jc.2016-1659

38. Dokras A, Sarwer DB, Allison KC, Milman L, Kris-Etherton PM, Kunselman AR, et al. Weight loss and lowering androgens predict improvements in healthrelated quality of life in women with PCOS. J Clin Endocrinol Metab (2016) 101(8):2966-74. doi:10.1210/jc.2016-1896

39. Renault KM, Nørgaard K, Nilas L, Carlsen EM, Cortes D, Pryds O, et al. The Treatment of Obese Pregnant Women (TOP) study: a randomized controlled trial of the effect of physical activity intervention assessed by pedometer with or without dietary intervention in obese pregnant women. Am J Obstet Gynecol (2014) 210(2):134.e1-9. doi:10.1016/j.ajog.2013.09.029

40. Phelan S, Phipps MG, Abrams B, Darroch F, Grantham K, Schaffner A, et al. Does behavioral intervention in pregnancy reduce postpartum weight retention? Twelve-month outcomes of the Fit for Delivery randomized trial. Am J Clin Nutr (2014) 99(2):302-11. doi:10.3945/ajcn.113.070151

41. Hawkins M, Hosker M, Marcus BH, Rosal MC, Braun B, Stanek EJ III, et al. A pregnancy lifestyle intervention to prevent gestational diabetes risk factors in overweight Hispanic women: a feasibility randomized controlled trial. Diabet Med (2015) 32(1):108-15. doi:10.1111/dme.12601

42. Dodd JM, Turnbull D, McPhee AJ, Deussen AR, Grivell RM, Yelland LN, et al. Antenatal lifestyle advice for women who are overweight or obese: LIMIT randomised trial. BMJ (2014) 348:g1285. doi:10.1136/bmj.g1285

43. Poston L, Briley AL, Barr S, Bell R, Croker H, Coxon K, et al. Developing a complex intervention for diet and activity behaviour change in obese pregnant women (the UPBEAT trial); assessment of behavioural change and process evaluation in a pilot randomised controlled trial. BMC Pregnancy Childbirth (2013) 13(1):148. doi:10.1186/1471-2393-13-148

44. Phelan S, Hagobian T, Brannen A, Hatley KE, Schaffner A, Muñoz-Christian K, et al. Effect of an Internet-based program on weight loss for low-income postpartum women: a randomized clinical trial. JAMA (2017) 317(23):2381-91. doi:10.1001/jama.2017.7119

45. Thompson FE, Kirkpatrick SI, Subar AF, Reedy J, Schap TE, Wilson MM, et al. The National Cancer Institute's dietary assessment primer: a resource for diet research. J Acad Nutr Diet (2015) 115(12):1986-95. doi:10.1016/j. jand.2015.08.016

Conflict of Interest Statement: The authors declare that the research was conducted in the absence of any commercial or financial relationships that could be construed as a potential conflict of interest.

Copyright $\odot 2017$ Chao, Srinivas, Studt, Diewald, Sarwer and Allison. This is an open-access article distributed under the terms of the Creative Commons Attribution License (CC BY). The use, distribution or reproduction in other forums is permitted, provided the original author(s) or licensor are credited and that the original publication in this journal is cited, in accordance with accepted academic practice. No use, distribution or reproduction is permitted which does not comply with these terms. 